\title{
Comparison results for exchangeable credit risk portfolios
}

\author{
Areski COUSIN, Jean-Paul LAURENT*
}

First Version: 11 September 2007

This version: 5 March 2008

\begin{abstract}
This paper is dedicated to the risk analysis of credit portfolios. Assuming that default indicators form an exchangeable sequence of Bernoulli random variables and as a consequence of de Finetti's theorem, default indicators are Binomial mixtures. We can characterize the supermodular order between two exchangeable Bernoulli random vectors in terms of the convex ordering of their corresponding mixture distributions. Thus we can proceed to some comparisons between stoploss premiums, CDO tranche premiums and convex risk measures on aggregate losses. This methodology provides a unified analysis of dependence for a number of CDO pricing models based on factor copulas, multivariate Poisson and structural approaches.
\end{abstract}

Key words: default risk, CDOs, factor copulas, multivariate Poisson, structural models, stochastic orders

PACS: C 31, G 13

\footnotetext{
* Jean-Paul Laurent is professor at ISFA Actuarial School, Université Claude Bernard Lyon 1 and a scientific consultant for BNP Paribas (laurent.jeanpaul@free.fr or laurent.jeanpaul@univlyon1.fr, http://laurent.jeanpaul.free.fr), 50 avenue Tony Garnier, 69007, LYON, FRANCE. Areski Cousin (areski.cousin@univ-lyon1.fr) is a PhD candidate at ISFA Actuarial School, Université Claude Bernard Lyon 1, 50 avenue Tony Garnier, 69007, LYON, FRANCE.

The authors thank M. Denuit, R. Elkamhi, M. Jeanblanc, C. Lefèvre, S. Loisel, F. Moraux, E. Marceau, A. Mc Neil, P. Picard, the participants of the December 2006 joint actuarial seminar of Lyon and Lausanne universities, the participants of the ENS Cachan money and banking seminar, the participants of the December 2007 Paris finance international meeting and the credit risk working group of Evry University for useful remarks. We also thank the referee for helpful comments. The usual disclaimer applies.
} 


\section{Introduction and Motivation}

Dependence modelling has recently been a subject of great interest in the finance and insurance fields. Actually, in most cases one cannot rely on the independence assumption and some form of modelling is required. Consequently, a number of models have been developed to capture dependencies between risks. Most of them belong to the class of individual risk models (or "bottom-up" approaches according to the credit derivatives terminology) which are well-suited to assess the impact of dependence structure on the riskiness of the portfolio.

Let us consider a pool of $n$ defaultable firms and denote by $\left(D_{1}^{t}, \ldots, D_{n}^{t}\right)$ the corresponding default indicator vector. $D_{i}^{t}$ is a Bernoulli random variable indicating whether name $i$ has defaulted before $t$. In case of default, name $i$ induces a loss amount $M_{i}$. The key quantity for the pricing and risk management of credit portfolios is the aggregate loss:

$$
L_{t}=\sum_{i=1}^{n} M_{i} D_{i}^{t} .
$$

For the sake of simplicity and because comparisons are established for a fixed horizon date, we voluntarily omit the suffix $t$ of $D_{i}^{t}$ in the following. We assume that $M_{1}, \ldots, M_{n}$ are independent random variables independent of $D_{i}, i=1 \ldots, n$. Moreover, we suppose that the dependence structure of $\left(D_{1}, \ldots, D_{n}\right)$ is governed by a correlation parameter $\theta$. We would like to assess the impact of $\theta$ on the riskiness of the total loss $L_{t}$ in the sense of, for example, an increase of stop-loss premiums or an increase of convex risk measures on $L_{t}$. More formally, for two default indicator vectors $\left(D_{1}, \ldots, D_{n}\right)$ and $\left(D_{1}^{*}, \ldots, D_{n}^{*}\right)$, we are looking for the conditions which leads to:

$$
E\left[\left(L_{t}-K\right)^{+}\right] \leq E\left[\left(L_{t}^{*}-K\right)^{+}\right] \text {for all } K \in \mathbb{R},
$$

or similarly to:

$$
\rho\left(L_{t}\right) \leq \rho\left(L_{t}^{*}\right) \text { for any convex risk measure } \rho \text {. }
$$

In order to ease the comparison procedure, we assume that default indicators are exchangeable Bernoulli random variables. When default indicators are part of an infinite sequence of random variables, de Finetti's theorem implies that $\left(D_{1}, \ldots, D_{n}\right)$ are Binomial mixtures. There exists a mixing random variable $\tilde{p}$ such that given $\tilde{p}, D_{1}, \ldots, D_{n}$ are conditionally independent. In the case of finite exchangeability, Jaynes (1986) stresses that the mixing measure may be signed and not necessarily unique. As far as applications are concerned, we will deal with Bernoulli vectors built from infinite exchangeable sequences and thus with Binomial mixtures.

From a risk analysis point of view, the exchangeability assumption is often implicitly used because it allows comparisons to be focussed on the dependence structure, moving away all possible marginal effects. Moreover, this representation result is a powerful tool since it allows to reduce the dependence structure to a single random factor $\tilde{p}$ leading to a more tractable analysis. We can ask at this stage whether some stochastic ordering results on $\left(D_{1}, \ldots, D_{n}\right)$ can be derived from $\tilde{p}$. Lefèvre and Utev (1996) showed that stop-loss ordering of $\tilde{p}$ yields stoploss ordering of $\sum D_{i}$, but this does not extend readily to non constant credit exposures across names. Denuit and Müller (2002) explored a multifactorial mixture framework and investigated the impact of the stochastic ordering of mixture distributions on the global dependence structure. 
Lately, we have witnessed a huge development of stochastic orders theory with applications to the actuarial field. The supermodular order has received a great attention since it immediately implies tractable comparison results on aggregate losses. Bäuerle and Müller (1998) and Müller (1997) investigated multivariate stochastic orders for several dependent risk models with applications to stop-loss ordering. Bäuerle and Müller (2005) specified the impact of some univariate stochastic orders on convex risk measures. Burgert and Rüschendorf (2006) extended the last paper to the multivariate case. The supermodular order has been treated specifically by Bäuerle (1997) and Müller and Scarsini (2000).

The modelling of dependence in the individual model has been investigated in several papers. Among others, Dhaene and Goovaerts (1997), Cossette et al. (2002) studied different orderings between two portfolios of dependent risks. Moreover, some comparison results in the credit risk case can be found in Burtschell et al. (2005) and Burtschell et al. (2007).

In this paper, we provide a complete characterization of the supermodular order for exchangeable Bernoulli random variables in terms of the convex ordering of mixture distributions. We use this result to suggest a new and general approach for analyzing the impact of dependence in default risk models.

The organization of the paper is as follows. In section 2, we recall the factor representation of exchangeable Bernoulli random vectors and provide some useful definitions and properties related to the theory of stochastic ordering. We then present our main results. In section 3, we analyze the impact of some dependence parameters in different credit risk models. We first consider additive factor copulas, a class of models including the Gaussian, the double $t$, the double NIG and the Variance Gamma copulas. We then study the class of Archimedean copulas and we end-up with the multivariate Poisson and the firm value models.

\section{Supermodular order for exchangeable Bernoulli variables}

\subsection{Exchangeability assumption and De Finetti's theorem}

Throughout the paper, we suppose that all random variables are defined on a common probability space $(\Omega, \mathcal{A}, P)$. Let us consider two exchangeable random vectors of Bernoulli variables $\mathbf{D}=$ $\left(D_{1}, \ldots, D_{n}\right)$ and $\mathbf{D}^{*}=\left(D_{1}^{*}, \ldots, D_{n}^{*}\right)$, i.e. the distributions of $\mathbf{D}$ and $\mathbf{D}^{*}$ are invariant for any permutations of their components. Kendall (1967) shows that a finite system of exchangeable events is equivalent to a random sampling scheme without replacement, where the number of items in the sampling has an arbitrary distribution. Extending de Finetti's theorem which involves infinite sequences of exchangeable Bernoulli variables, Jaynes (1986) shows that the distributions of $\mathbf{D}$ and $\mathbf{D}^{*}$ admit a factor representation in terms of a signed mixture measure:

Proposition 2.1 de Finetti's representation of exchangeable Bernoulli vectors Let $\left(D_{1}, \ldots, D_{n}\right)$ and $\left(D_{1}^{*}, \ldots, D_{n}^{*}\right)$ be two exchangeable Bernoulli random vectors. Then, there 
exists a signed mixture measure $\nu$ on $([0,1], \mathcal{B}([0,1]))$ (not necessarily unique) such that:

$$
P\left(D_{1}=d_{1}, \ldots, D_{n}=d_{n}\right)=\int p^{x}(1-p)^{n-x} \nu(d p),
$$

where $d_{i}=0,1, i=1, \ldots, n, x=\sum_{i=1}^{n} d_{i}$. Similarly, there exists a signed mixture measure $\nu^{*}$ on $([0,1], \mathcal{B}([0,1]))$ (not necessarily unique) such that:

$$
P\left(D_{1}^{*}=d_{1}, \ldots, D_{n}^{*}=d_{n}\right)=\int p^{x}(1-p)^{n-x} \nu^{*}(d p)
$$

In Jaynes (1986) approach, a signed density is expressed in the Legendre orthogonal polynomial basis. On numerical grounds, Kendall (1967) results can be used to improve the computation of this signed density.

When for all $n \geq 1, D_{1}, \ldots, D_{n}$ and $D_{1}^{*}, \ldots, D_{n}^{*}$ are subsets of exchangeable Bernoulli sequences $\left(D_{1}, \ldots, D_{n}, \ldots\right)$ and $\left(D_{1}^{*}, \ldots, D_{n}^{*}, \ldots\right)$, then, as a consequence of de Finetti's theorem, $\nu$ and $\nu^{*}$ must be probability measures. In that case, representations 2.1 and 2.2 are unique and there are two underlying factors $\tilde{p}$ and $\tilde{p}^{*}$ distributed as (resp.) $\nu$ and $\nu^{*}$ such that $D_{1}, \ldots, D_{n}$ are independent given $\tilde{p}$ and $D_{1}^{*}, \ldots, D_{n}^{*}$ are independent given $\tilde{p}^{*} . \tilde{p}$ and $\tilde{p}^{*}$ are characterized by:

$$
\frac{1}{n} \sum_{i=1}^{n} D_{i} \stackrel{a . s}{\longrightarrow} \tilde{p}, \frac{1}{n} \sum_{i=1}^{n} D_{i}^{*} \stackrel{a . s}{\longrightarrow} \tilde{p}^{*} \quad \text { as } n \rightarrow \infty .
$$

In this paper, we analyze the influence of the mixture measure $\nu$ onto the dependence structure of $\mathbf{D}$. Loosely speaking, we will show that comparing $\nu$ with $\nu^{*}$ is equivalent to comparing $\mathbf{D}$ with $\mathbf{D}^{*}$. Most of the time, we will deal with Bernoulli vectors built from infinite exchangeable sequences so that the study will be focused on mixture probabilities. But, when a generalization is possible, signed mixture measures will be used to perform the comparison results. To do so, let us now recall some definitions and properties related to stochastic ordering that will be used later. For more details related to stochastic ordering theory, the reader is referred to the seminal books of Denuit et al. (2005), Müller and Stoyan (2002) and Shaked and Shanthikumar (1994).

\subsection{Some stochastic ordering results}

We first present a generalization of the convex order to signed measures.

Definition 2.2 Let $\nu$ and $\nu^{*}$ be two signed measures. We say that:

(1) $\nu$ is less than $\nu^{*}$ in convex order (written $\left.\nu \leq_{c x} \nu^{*}\right)$ if $\int u(p) \nu(d p) \leq \int u(p) \nu^{*}(d p)$ for all real convex functions $u$ such that the integrals exist.

(2) $\nu$ is less than $\nu^{*}$ in increasing convex order (written $\nu \leq_{i c x} \nu^{*}$ ) if $\int u(p) \nu(d p) \leq$ $\int u(p) \nu^{*}(d p)$ for all increasing convex functions $u$ such that the integrals exist. 
Unless otherwise stated, we assume in the sequel that $\nu$ and $\nu^{*}$ are probability measures and we denote by $F$ (resp. $F^{*}$ ) the distribution function associated with $\nu$ (resp. $\nu^{*}$ ). For $p \in[0,1]$, $F(p)=\nu([0, p])$ and $F^{*}(p)=\nu^{*}([0, p])$. Consider two random variables $\tilde{p}$ and $\tilde{p}^{*}$ distributed as (resp.) $F$ and $F^{*}$. We will indifferently denote by $\nu \leq_{\mathcal{F}} \nu^{*}, \tilde{p} \leq_{\mathcal{F}} \tilde{p}^{*}$ or $F \leq_{\mathcal{F}} F^{*}$ the fact that $\nu$ is less than $\nu^{*}$ with respect to the $\mathcal{F}$-order.

Let us first remark that the convex ordering and the increasing convex ordering of distribution functions are captured by Definition 2.2 as probability measures are particular signed measures. But, for the sake of clarity and presentation, we choose to recall the stochastic versions of these definitions. We then expose other useful stochastic orders and related properties.

Definition 2.3 Let $F$ and $F^{*}$ be two distribution functions. We say that:

(1) $F$ is less than $F^{*}$ in convex order (written $\left.F \leq_{c x} F^{*}\right)$ if $\int u(p) d F(p) \leq \int u(p) d F^{*}(p)$ for all real convex functions $u$ such that the expectations exist.

(2) $F$ is less than $F^{*}$ in increasing convex order (written $F \leq_{i c x} F^{*}$ ) if $\int u(p) d F(p) \leq$ $\int u(p) d F^{*}(p)$ for all increasing convex functions $u$ such that the expectations exist.

(3) $F$ is less than $F^{*}$ in the stop loss order (written $\left.F \leq_{s l} F^{*}\right)$ if $\int(p-t)^{+} d F(p) \leq \int(p-$ $t)^{+} d F^{*}(p)$, for all $t \in \mathbb{R}$ such that the expectations exist.

(4) $F$ is said to be less dangerous than $F^{*}$ (written $F \leq_{D} F^{*}$ ) if there is $p_{0}$ such that $F(p) \leq F^{*}(p)$ for all $p<p_{0}, F(p) \geq F^{*}(p)$ for all $p \geq p_{0}$ and if $\int p d F(p) \leq \int p d F^{*}(p)$.

Strassen's theorem provides a characterization of the convex order:

Theorem 2.4 Let us consider two distribution functions $F$ and $F^{*}$ associated with finite means ${ }^{1}$. The following conditions are equivalent:

(1) $F \leq_{c x} F^{*}$,

(2) there exists two real random variables $\tilde{p}$ and $\tilde{p}^{*}$ with distribution functions $F$ and $F^{*}$, such that $\tilde{p}^{*}=\tilde{p}+u$ and $E[u \mid \tilde{p}]=0$.

In other words, $\tilde{p}^{*}$ is obtained from $\tilde{p}$ by adding some noise $u$. We also quote the following result from Müller and Stoyan (2002) textbook:

Proposition 2.5 Let $F$ and $F^{*}$ be two distribution functions. We have:

(1) $F \leq_{s l} F^{*} \Leftrightarrow F \leq_{i c x} F^{*}$

(2) $F \leq_{D} F^{*} \Rightarrow F \leq_{i c x} F^{*}$

We recall the following result that relates convex and increasing convex orders:

$\overline{1 \int_{\mathbb{R}} \mid p} \mid d F(p)<\infty$ and $\int_{\mathbb{R}}|p| d F^{*}(p)<\infty$. 
Proposition 2.6 The following statements are equivalent:

(1) $F \leq_{c x} F^{*}$,

(2) $F \leq_{i c x} F^{*}$ and $\int p d F(p)=\int p d F^{*}(p)$.

Proof: see Müller and Stoyan (2002), Theorem 1.5.3.

Since $P\left(D_{i}=1\right)=E\left[D_{i}\right]=\int_{0}^{1} p d F(p), i=1, \ldots, n, F \leq_{c x} F^{*}$ implies that $D_{1}, \ldots, D_{n}, D_{1}^{*}, \ldots, D_{n}^{*}$ have the same distribution.

Definition 2.7 A function $f: \mathbb{R}^{n} \rightarrow \mathbb{R}$ is said to be supermodular if

$$
\begin{aligned}
& f\left(x_{1}, \ldots, x_{i}+\varepsilon, \ldots, x_{j}+\delta, \ldots, x_{n}\right)-f\left(x_{1}, \ldots, x_{i}+\varepsilon, \ldots, x_{j}, \ldots, x_{n}\right) \\
& \geq \quad f\left(x_{1}, \ldots, x_{i}, \ldots, x_{j}+\delta, \ldots, x_{n}\right)-f\left(x_{1}, \ldots, x_{i}, \ldots, x_{j}, \ldots, x_{n}\right)
\end{aligned}
$$

holds for all $x \in \mathbb{R}^{n}, 1 \leq i<j \leq n$ and $\varepsilon, \delta>0$. If in addition (2.4) holds for $i=j$, then $f$ is said to be directionally convex.

Suppose that $x_{i}$ indicates with 1 or 0 whether a default of name $i$ occurs or not and that $f$ provides the induced total loss. Assume that two default cases at date $t$ can occur. In the first one, $m$ defaults have occurred while in the second case $m+1$ defaults have occurred (the same $m$ defaults plus another one). Then, $f$ is supermodular if the impact of a fresh new default is worse in the latter case than in the former.

Let us now state the definition of some multivariate stochastic orders related to the supermodular class of functions.

Definition 2.8 Let $\mathbf{D}=\left(D_{1}, \ldots, D_{n}\right)$ and $\mathbf{D}^{*}=\left(D_{1}^{*}, \ldots, D_{n}^{*}\right)$ be two random vectors.

(1) $\mathbf{D}$ is said to be smaller than $\mathbf{D}^{*}$ in the supermodular order (written $\mathbf{D} \leq_{s m} \mathbf{D}^{*}$ ) if $E[f(\mathbf{D})] \leq E\left[f\left(\mathbf{D}^{*}\right)\right]$, for all supermodular functions $f$ such that the expectation exists.

(2) $\mathbf{D}$ is said to be smaller than $\mathbf{D}^{*}$ in the increasing supermodular order (written $\mathbf{D} \leq \leq_{i s m}$ $\left.\mathbf{D}^{*}\right)$ if $E[f(\mathbf{D})] \leq E\left[f\left(\mathbf{D}^{*}\right)\right]$, for all increasing and supermodular functions $f$ such that the expectation exists.

(3) $\mathbf{D}$ is said to be smaller than $\mathbf{D}^{*}$ in the directionally convex order (written $\mathbf{D} \leq{ }_{d c x} \mathbf{D}^{*}$ ) if $E[f(\mathbf{D})] \leq E\left[f\left(\mathbf{D}^{*}\right)\right]$, for all directionally convex functions $f$ such that the expectation exists.

Even if the supermodular order does not look quite intuitive at first sight, it leads to some useful results on the risk analysis of aggregate losses. As an example, Müller (1997) shows some comparisons of stop-loss premiums: 
Proposition 2.9 Let $\left(D_{1}, \ldots, D_{n}\right)$ and $\left(D_{1}^{*}, \ldots, D_{n}^{*}\right)$ be two random vectors. Let $M_{1}, \ldots, M_{n}$ be some non negative independent nominal exposures independent of $D_{1}, \ldots, D_{n}, D_{1}^{*}, \ldots, D_{n}^{*}$ and $L=\sum_{i=1}^{n} M_{i} D_{i}$ and $L^{*}=\sum_{i=1}^{n} M_{i} D_{i}^{*}$. Then,

$$
\left(D_{1}, \ldots, D_{n}\right) \leq_{s m}\left(D_{1}^{*}, \ldots, D_{n}^{*}\right) \Rightarrow L \leq_{s l} L^{*} .
$$

Moreover, the supermodular order is consistent with ordering of law-invariant and convex risk measures on the aggregate loss. In particular, law invariant coherent risk measures such as the Expected Shortfall or Wang transform risk measure are concerned. This can be derived from the following result of Bäuerle and Müller (2005):

Proposition 2.10 Consider two random variables $L$ and $L^{*}$. Then,

$$
L \leq_{s l} L^{*} \Rightarrow \rho(L) \leq \rho\left(L^{*}\right)
$$

for any law-invariant and convex risk measure $\rho$.

In the field of stochastic ordering, not so much research has been devoted to analyze how the mixture distribution impacts the global risk. For exchangeable Bernoulli random variables, Lefèvre and Utev (1996) have shown that if $F$ and $F^{*}$ are ordered in the sense of the increasing convex order, then the corresponding aggregate claims $L_{n}=\sum_{i=1}^{n} D_{i}$ and $L_{n}^{*}=\sum_{i=1}^{n} D_{i}^{*}$ are ordered in the same way. Specifically, Corollary 3.7 in Lefèvre and Utev (1996) is the s-convex generalization of this well-known result (also mentioned in Bäuerle and Müller (1998)):

$$
F \leq_{i c x} F^{*} \Rightarrow L_{n} \leq_{s l} L_{n}^{*}
$$

Proof: It is a direct consequence of example 6.A.2 p.172 in Shaked and Shanthikumar (1994).

In the following, this result will be generalized to compare exchangeable Bernoulli random vectors with respect to the supermodular order.

\subsection{Main result}

\section{Theorem 2.11 Supermodular order for exchangeable Bernoulli vectors}

Consider two exchangeable random vectors of Bernoulli variables $\left(D_{1}, \ldots, D_{n}\right)$ and $\left(D_{1}^{*}, \ldots, D_{n}^{*}\right)$ and denote by $\nu$ and $\nu^{*}$ two associated mixture measures. Then,

$$
\nu \leq_{c x} \nu^{*} \Rightarrow\left(D_{1}, \ldots, D_{n}\right) \leq_{s m}\left(D_{1}^{*}, \ldots, D_{n}^{*}\right),
$$

and

$$
\nu \leq_{i c x} \nu^{*} \Rightarrow\left(D_{1}, \ldots, D_{n}\right) \leq_{i s m}\left(D_{1}^{*}, \ldots, D_{n}^{*}\right) .
$$

Proof: let $f$ be a supermodular function. Following Proposition 2.1,

$$
E\left[f\left(D_{1}, \ldots, D_{n}\right)\right]=\sum_{\left(d_{1}, \ldots, d_{n}\right) \in\{0,1\}^{n}} f\left(d_{1}, \ldots, d_{n}\right) P\left(D_{1}=d_{1}, \ldots, D_{n}=d_{n}\right)
$$


can be written as:

$$
\int_{0}^{1} \sum_{\left(d_{1}, \ldots, d_{n}\right) \in\{0,1\}^{n}} f\left(d_{1}, \ldots, d_{n}\right) p^{d_{1}+\cdots+d_{n}}(1-p)^{n-\left(d_{1}+\cdots+d_{n}\right)} \nu(d p) .
$$

Let us consider the function $v:[0,1]^{n} \rightarrow \mathbb{R}$ defined by:

$$
v\left(p_{1}, \ldots, p_{n}\right)=\sum_{\left(d_{1}, \ldots, d_{n}\right) \in\{0,1\}^{n}} f\left(d_{1}, \ldots, d_{n}\right) p_{1}^{d_{1}}\left(1-p_{1}\right)^{1-d_{1}} \ldots p_{n}^{d_{n}}\left(1-p_{n}\right)^{1-d_{n}}
$$

Let us show that $v$ is supermodular. We can re-write $v\left(p_{1}, \ldots, p_{n}\right)$ as:

$$
\begin{gathered}
p_{1} \sum_{\left(d_{2}, \ldots, d_{n}\right) \in\{0,1\}^{n-1}} f\left(1, d_{2}, \ldots, d_{n}\right) p_{2}^{d_{2}}\left(1-p_{2}\right)^{1-d_{2}} \ldots p_{n}^{d_{n}}\left(1-p_{n}\right)^{1-d_{n}} \\
+\left(1-p_{1}\right) \sum_{\left(d_{2}, \ldots, d_{n}\right) \in\{0,1\}^{n-1}} f\left(0, d_{2}, \ldots, d_{n}\right) p_{2}^{d_{2}}\left(1-p_{2}\right)^{1-d_{2}} \ldots p_{n}^{d_{n}}\left(1-p_{n}\right)^{1-d_{n}} .
\end{gathered}
$$

Thus,

$$
\frac{\partial v}{\partial p_{1}}=\sum_{\left(d_{2}, \ldots, d_{n}\right) \in\{0,1\}^{n-1}}\left(f\left(1, d_{2}, \ldots\right)-f\left(0, d_{2}, \ldots\right)\right) p_{2}^{d_{2}}\left(1-p_{2}\right)^{1-d_{2}} \ldots p_{n}^{d_{n}}\left(1-p_{n}\right)^{1-d_{n}} .
$$

Similarly, we can write $\frac{\partial^{2} v}{\partial p_{1} \partial p_{2}}$ as:

$\sum_{\left(d_{3}, \ldots, d_{n}\right) \in\{0,1\}^{n-2}}(f(1,1, \ldots)-f(1,0, \ldots)-f(0,1, \ldots)+f(0,0, \ldots)) p_{3}^{d_{3}}\left(1-p_{3}\right)^{1-d_{3}} \ldots p_{n}^{d_{n}}\left(1-p_{n}\right)^{1-d_{n}}$,

which is non negative since $f$ is supermodular. We can proceed similarly for any pair $i, j, 1 \leq$ $i<j \leq n$. This shows that $v$ is supermodular. Let us also remark that $\frac{\partial^{2} v}{\partial p_{i}^{2}}=0, i=1, \ldots, n$. As a consequence, the Hessian matrix associated with $v$ has non negative components. This implies that the real function $p \in \mathbb{R} \rightarrow u(p)=v(p, \ldots, p)$ is convex because the second derivative of $u$ is equal to the sum of the components of the Hessian matrix of $v$. From (2.10), we can write $E\left[f\left(D_{1}, \ldots, D_{n}\right)\right]$ as $\int u(p) \nu(d p)$. Since $u$ is convex and $\nu \leq_{c x} \nu^{*}$, we have $\int u(p) \nu(d p) \leq$ $\int u(p) \nu^{*}(d p)$, where the former integral is also equal to $E\left[f\left(D_{1}^{*}, \ldots, D_{n}^{*}\right)\right]$. If, in addition, $f$ is supposed to be increasing, then the partial derivatives of $v, \frac{\partial v}{\partial p_{i}}, i=1, \ldots, n$ are non-negative. Thus, the function $u$ is non decreasing and convex and we obtain (2.9) by the same line of reasoning.

Let us remark that our result can be derived from Proposition 4.1 in Denuit and Frostig (2007). Their proof goes into the same lines as ours and was shown independently.

We now present the reverse implication which requires Bernoulli vectors to be ordered in the sense of the supermodular order for any dimension. 
Theorem 2.12 Let $D_{1}, \ldots, D_{n}, \ldots$ and $D_{1}^{*}, \ldots, D_{n}^{*}, \ldots$ be two exchangeable sequences of Bernoulli variables. We denote by $F\left(\right.$ resp. $\left.F^{*}\right)$ the distribution function associated with the mixing measure. Then,

$$
\left(D_{1}, \ldots, D_{n}\right) \leq_{s m}\left(D_{1}^{*}, \ldots, D_{n}^{*}\right), \forall n \in \mathbb{N} \Rightarrow F \leq_{c x} F^{*}
$$

Proof: let us denote by $Y_{n}:=\frac{1}{n} \sum_{i=1}^{n} D_{i}$ and $Y_{n}^{*}:=\frac{1}{n} \sum_{i=1}^{n} D_{i}^{*}$. Let $t \in \mathbb{R}^{+} .\left(Y_{n}-t\right)^{+} \leq 1$ and $\left(Y_{n}^{*}-t\right)^{+} \leq 1$. From de Finetti's theorem, $Y_{n} \stackrel{a . s .}{\rightarrow} Y_{\infty}$ where $Y_{\infty}$ has distribution function $F$. From dominated convergence theorem, $\lim _{n \rightarrow \infty} E\left[\left(Y_{n}-t\right)^{+}\right]=E\left[\left(Y_{\infty}-t\right)^{+}\right]$. Similarly, $\lim _{n \rightarrow \infty} E\left[\left(Y_{n}^{*}-t\right)^{+}\right]=E\left[\left(Y_{\infty}^{*}-t\right)^{+}\right]$. Since $\left(D_{1}, \ldots, D_{n}\right) \leq_{s m}\left(D_{1}^{*}, \ldots, D_{n}^{*}\right), Y_{n} \leq_{s l} Y_{n}^{*}$. Thus, $E\left[\left(Y_{n}-t\right)^{+}\right] \leq E\left[\left(Y_{n}^{*}-t\right)^{+}\right], \forall n \in \mathbb{N}$. This yields $E\left[\left(Y_{\infty}-t\right)^{+}\right] \leq E\left[\left(Y_{\infty}^{*}-t\right)^{+}\right]$which shows that $F \leq_{i c x} F^{*}$. Eventually, since $\left(D_{1}, \ldots, D_{n}\right) \leq_{s m}\left(D_{1}^{*}, \ldots, D_{n}^{*}\right), D_{1}$ and $D_{1}^{*}$ have the same marginal distribution. $P\left(D_{1}=1\right)=E\left[D_{1}\right]=\int p d F(p)$. Similarly, $P\left(D_{1}^{*}=1\right)=\int p d F^{*}(p)$, thus $\int p d F(p)=\int p d F^{*}(p)$, which eventually shows that $F \leq_{c x} F^{*}$.

Suppose now that $\left(D_{1}, \ldots, D_{n}\right)$ and $\left(D_{1}^{*}, \ldots, D_{n}^{*}\right)$ are exchangeable Bernoulli vectors which may not necessarily be subsets of exchangeable random sequences. In view of Proposition 2.1, there may exist several mixture measures associated with de Finetti's representation. We may then ask whether there exist some mixture measures $\nu_{n}$ and $\nu_{n}^{*}$ such that:

$$
\left(D_{1}, \ldots, D_{n}\right) \leq_{s m}\left(D_{1}^{*}, \ldots, D_{n}^{*}\right) \Rightarrow \nu_{n} \leq_{c x} \nu_{n}^{*}
$$

The answer is not that simple. Let us consider a zero mean, unit variance Gaussian vector $\left(V_{1}, \ldots, V_{n}\right)$. Following the approach of Jaynes (1986), we used Legendre orthogonal polynomials to construct the mixture measure of the exchangeable random vector $\mathbf{D}=\left(1_{V_{1} \leq v_{0}}, \ldots, 1_{V_{n} \leq v_{0}}\right)$ where $v_{0}$ is a real number. Exchangeability assumption requires that pairwise correlations are equal, say to $\rho$. We assume that the mixture measure admits a density function $g$, i.e. $\nu(d p)=$ $g(p) d p$. Starting from the joint distribution of $\mathbf{D}$, we numerically computed $g$ and stop-loss premiums on $g\left(\int_{0}^{1}(p-k)^{+} g(p) d p, k \in[0,1]\right)$ for $n=3$ and $n=4$ and for several admissible values of $\rho$ (see Fig. 1 and Fig. 2).
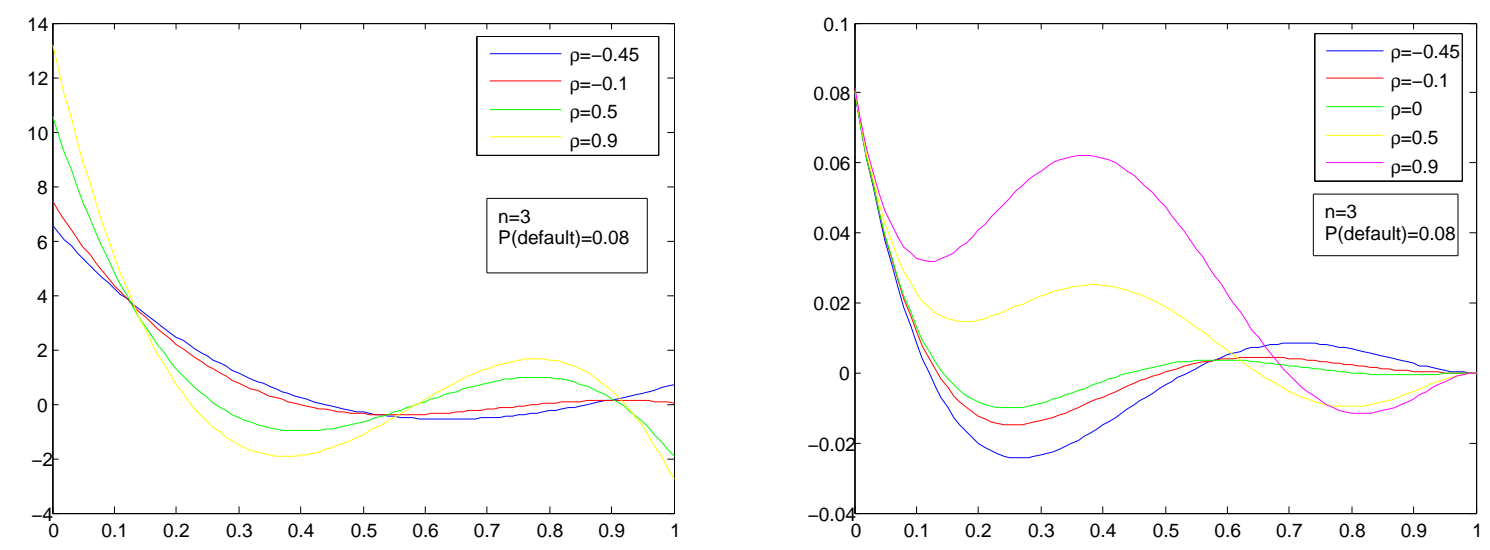

Fig. 1. Mixing densities (left) and corresponding stop loss premiums (right) for $n=3$

It is well known that when the correlation parameter $\rho$ increases, the Gaussian vector $\left(V_{1}, \ldots, V_{n}\right)$ increases in the sense of the supermodular order. As this order is closed by monotone transformations, the Bernoulli vector $\mathbf{D}$ consequently increases. But, as we can see in Fig. 1 and Fig. 2, 

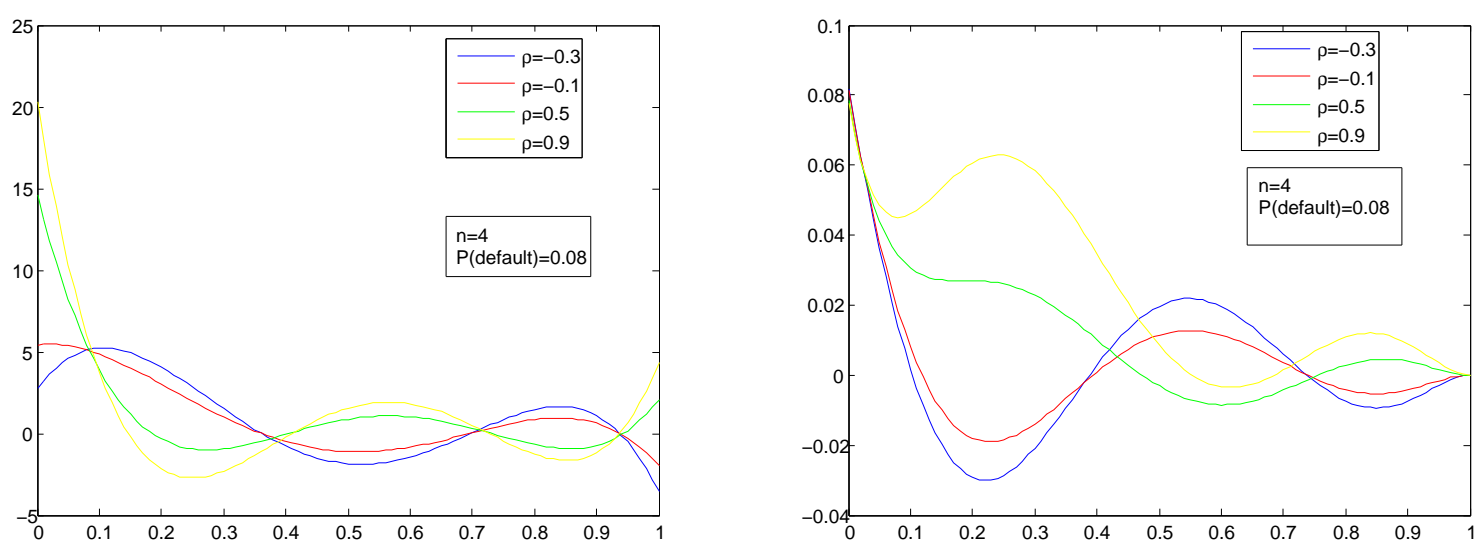

Fig. 2. Mixing densities (left) and corresponding stop loss premiums (right) for $n=4$

stop-loss premiums on mixture measures do not increase for all retention levels when $\rho$ increases. So, at least for $n=3$ and $n=4$, (2.12) does not hold for the Legendre polynomial construction of mixture measures.

\section{Applications to credit risk management}

The credit derivatives market has witnessed a huge development, growing both in size and in complexity. Many products are related to default events within a reference credit portfolio. The most popular multiname credit derivatives are the basket credit default swaps (Basket CDS) and the collateralized debt obligations (CDOs). They can both be seen as insurance contracts against the default of one or several entities. Basket CDS cover losses induced by defaults of a group of names within a reference portfolio whereas CDOs payments are related to cumulative losses occurring in a certain tranche $[\alpha \%, \beta \%]$ of the total notional (see Laurent and Gregory (2005) for more details about the pricing of basket CDS and CDOs).

In the following, we will review a number of default risk models: additive factor copulas models (including the Gaussian, the double-t, the double NIG and the Variance Gamma cases), Archimedean copula, multivariate Poisson and eventually structural models. For each of them, we will compute the mixture distribution and therefore apply Theorem 2.11 to analyze the model parameters impact onto the supermodular ordering of default indicator vectors, thus extending some results of Burtschell et al. (2005) and Burtschell et al. (2007). Additional applications can be found in Denuit and Frostig (2007).

\subsection{Additive factor copula models}

In the additive factor copula approach, the dependence structure of default times is driven by some latent variables $V_{1}, \ldots, V_{n}$ with the following dependence structure:

$$
V_{i}=\rho V+\sqrt{1-\rho^{2}} \bar{V}_{i}, \quad i=1, \ldots, n .
$$


We assume that $V$ and $\bar{V}_{i}, i=1, \ldots, n$ are independent random variables and $0 \leq \rho \leq 1$. Moreover, we denote by $H$ the cumulative density function (cdf) of $V$, by $\bar{H}$ the common cdf of $\bar{V}_{i}, i=1 \ldots n$ and by $H_{\rho}$ the common cdf of $V_{i}, i=1 \ldots n$. Let us remark that, in our framework, while $H$ and $\bar{H}$ do not depend on the parameter $\rho$, the resulting convolution cdf $H_{\rho}$ generally does.

The exchangeability assumption requires that all default times $\tau_{i}$ have the same cdf, say $G$. The default times are then expressed as:

$$
\tau_{i}=G^{-1}\left(H_{\rho}\left(V_{i}\right)\right) \quad i=1, \ldots, n,
$$

where $G^{-1}$ denote the generalized inverse of $G$.

We aim at analyzing the influence of the dependence parameter $\rho$ on supermodular comparisons of default indicator vectors at a given horizon date $t$. Denote by $\bar{p}=G(t)$ the common default probability at time $t$ and by:

$$
D_{i}=1_{\left\{\tau_{i} \leq t\right\}}=1_{\left\{H_{\rho}\left(V_{i}\right) \leq \bar{p}\right\}}, \quad i=1 \ldots n .
$$

$\left(D_{1}, \ldots, D_{n}\right)$ is an exchangeable Bernoulli random vector and $D_{1}, \ldots, D_{n}$ are independent given the common factor $V$.

Thanks to remark (2.3), the mixture probability associated with $\left(D_{1}, \ldots, D_{n}\right)$ corresponds to the conditional default probability:

$$
\frac{1}{n} \sum_{i=1}^{n} D_{i} \stackrel{a . s}{\longrightarrow} \tilde{p}=E\left[D_{i} \mid V\right]=P\left(\tau_{i} \leq t \mid V\right) \quad \text { as } n \rightarrow \infty .
$$

We have thus:

$$
\tilde{p}=\bar{H}\left(\frac{H_{\rho}^{-1}(\bar{p})-\rho V}{\sqrt{1-\rho^{2}}}\right) .
$$

The following proposition states that when the dependence parameter increases from $\rho$ to $\rho^{*}$, the associated mixture probability $\tilde{p}$ is less dangerous than $\tilde{p}^{*}$.

Proposition 3.1 Assume that $\bar{V}_{i}, i=1 \ldots n$ are continuous random variables of range $\mathbb{R}$. If $\rho \leq \rho^{*}$, then

$$
\tilde{p}=\bar{H}\left(\frac{H_{\rho}^{-1}(\bar{p})-\rho V}{\sqrt{1-\rho^{2}}}\right) \leq_{D} \tilde{p}^{*}=\bar{H}\left(\frac{H_{\rho^{*}}^{-1}(\bar{p})-\rho^{*} V}{\sqrt{1-\rho^{* 2}}}\right)
$$

Proof: Let us note $F$ and $F^{*}$ the cdf of (resp.) $\tilde{p}$ and $\tilde{p}^{*}$. In view of Definition 2.3, we have to show that the difference $F-F^{*}$ alternates in sign from negative to positive once. But $F$ can be expressed as:

$$
F(p)=1-H\left(\frac{H_{\rho}^{-1}(\bar{p})-\sqrt{1-\rho^{2}} \bar{H}^{-1}(p)}{\rho}\right)
$$

As $H$ is increasing and does not depend on $\rho, F(p)-F^{*}(p)$ has the sign of:

$$
h(p)=\left(\rho^{*} \sqrt{1-\rho^{2}}-\rho \sqrt{1-\rho^{* 2}}\right) \bar{H}^{-1}(p)+\rho H_{\rho^{*}}^{-1}(\bar{p})-\rho^{*} H_{\rho}^{-1}(\bar{p})=A \bar{H}^{-1}(p)-B
$$


Since $A \geq 0, h$ is increasing in $p$. Moreover, $\lim _{p \rightarrow 0} h(p)=-\infty$ and $\lim _{p \rightarrow 1} h(p)=+\infty$ so that there exists $0 \leq p_{0} \leq 1$ such that $h(p) \leq 0$ for all $p<p_{0}$ and $h(p) \geq 0$ for all $p \geq p_{0}$.

Corollary 3.2 Assume that $\bar{V}_{i}, i=1 \ldots n$ are continuous random variables of range $\mathbb{R}$. If $\rho \leq \rho^{*}$, then:

$$
\tilde{p} \leq_{c x} \tilde{p}^{*}
$$

and with Theorem 2.11,

$$
\left(D_{1}, \ldots, D_{n}\right) \leq_{s m}\left(D_{1}^{*}, \ldots, D_{n}^{*}\right)
$$

Proof: In view of Propositions 3.1 and $E[\tilde{p}]=E\left[\tilde{p}^{*}\right]=\bar{p},(3.5)$ derives from Proposition 2.5 and 2.6 .

The previous framework includes the Gaussian copula, the double- $t$ copula (Hull and White (2004)), the NIG copula (Guegan and Houdain (2005)), the double-NIG copula (Kalemanova et al. (2007)) and the double Variance Gamma copula (Moosbrucker (2006)).

\subsection{One factor Archimedean copulas}

Archimedean copulas have been widely used in credit risk modelling: They represent an alternative to the Gaussian copula. In most cases, there exists an effective and tractable way of generating random vectors with this dependence structure. Eventually, Archimedean copulas are exchangeable.

Definition 3.3 $C:[0,1]^{n} \rightarrow[0,1]$ is an $\varphi$-Archimedean copula if:

$$
\forall\left(u_{1}, \ldots, u_{n}\right) \in[0,1]^{n}, C\left(u_{1}, \ldots, u_{n}\right)=\varphi^{-1}\left(\varphi\left(u_{1}\right)+\ldots+\varphi\left(u_{n}\right)\right),
$$

where the generating function $\varphi:[0,1] \rightarrow[0,+\infty]$ is a continuous strictly decreasing function such that $\varphi^{-1}$ is the Laplace transform of a positive random variable $V$, i.e. $\varphi^{-1}(t)=$ $E[\exp (-t V)], t \in[0,+\infty]$.

Conditions imposed on $\varphi$ are quite strong but yields an admissible distribution function for all dimensions $n \geq 2$. In fact, it is possible to consider weaker conditions on $\varphi$ in order to obtain admissible distribution functions up to a particular dimension $n$ (see Nelsen (1999) for more details).

Marshall and Olkin (1988) have provided a well-known simulation algorithm which we recall below.

Proposition 3.4 Let $V$ be a positive random variable with Laplace transform $\varphi^{-1}$ and $U_{1}, \ldots, U_{n}$ be independent uniformly distributed random variables independent of $V$. If $V_{1}, \ldots, V_{n}$ are defined by:

$$
V_{i}=\varphi^{-1}\left(-\frac{\ln U_{i}}{V}\right), i=1 \ldots n
$$


then $\left(V_{1}, \ldots, V_{n}\right)$ is distributed according to a $\varphi$-Archimedean copula.

We now build a default time model based on the previous dependence structure. The exchangeability assumption requires that all default times $\tau_{i}$ have the same cdf, say $G$. Let us define the latent variables $V_{i}, i=1 \ldots n$ as in Proposition 3.4. The default times are then expressed as:

$$
\tau_{i}=G^{-1}\left(V_{i}\right) \quad i=1, \ldots, n,
$$

where $G^{-1}$ denote the generalized inverse of $G$.

We aim at analyzing the influence of the generating function $\varphi$ on comparisons of default indicator vectors with respect to the supermodular order. Furthermore, comparisons are established at a fixed horizon $t$.

If we denote by $\bar{p}=G(t)$ the default probability at time $t$ and by $D_{i}=1_{\left\{\tau_{i} \leq t\right\}}=1_{\left\{V_{i} \leq \bar{p}\right\}}$, $i=1 \ldots n$, the corresponding default indicators, then $\left(D_{1}, \ldots, D_{n}\right)$ is an exchangeable Bernoulli random vector. Besides, $D_{1}, \ldots, D_{n}$ are independent given the common factor $V$.

Thanks to remark (2.3), the mixture probability $\tilde{p}$ associated with $\left(D_{1}, \ldots, D_{n}\right)$ is equal to the conditional default probability $P\left(\tau_{i} \leq t \mid V\right)$ :

$$
\tilde{p}=\exp \{-\varphi(\bar{p}) V)\}
$$

Let us consider another default indicator vector $\left(D_{1}^{*}, \ldots, D_{n}^{*}\right)$ associated with a $\psi$-Archimedean copula. Then, there is a condition on $\varphi$ and $\psi$ which allows to compare the mixture probabilities $\tilde{p}$ and $\tilde{p}^{*}$ with respect to the convex order.

Proposition 3.5 Let $V$ (resp. $V^{*}$ ) be a positive random variable and denote by $\varphi^{-1}$ (resp. $\psi^{-1}$ ) its Laplace transform. Define by $\mathscr{L}_{\infty}^{*}$ the set of all non decreasing functions $f: \mathbb{R}^{+} \rightarrow \mathbb{R}^{+}$with alternate signs for the derivatives:

$$
\mathscr{L}_{\infty}^{*}=\left\{f: \mathbb{R}^{+} \rightarrow \mathbb{R}^{+} \mid(-1)^{n-1} f^{(n)} \geq 0 \forall n \geq 1\right\} .
$$

Let us consider $\tilde{p}=\exp (-\varphi(\bar{p}) V)$ and $\tilde{p}^{*}=\exp \left(-\psi(\bar{p}) V^{*}\right)$, the corresponding mixture probabilities. If $\varphi \circ \psi^{-1} \in \mathscr{L}_{\infty}^{*}$, then $\tilde{p} \leq_{c x} \tilde{p}^{*}$ for all $\bar{p}$ in $[0,1]$.

Proof: Suppose that $\varphi \circ \psi^{-1} \in \mathscr{L}_{\infty}^{*}$. Then in view of Theorem 3.1 of Wei and Hu (2002), for any dimension $n$, the Archimedean copula generated by $\psi$ is greater than the Archimedean copula generated by $\varphi$ in the sense of the supermodular order. In other words, $\left(V_{1}, \ldots, V_{n}\right) \leq_{s m}$ $\left(V_{1}^{*}, \ldots, V_{n}^{*}\right)$ holds for all $n$ whenever $\left(V_{1}, \ldots, V_{n}\right)$ (resp. $\left.\left(V_{1}^{*}, \ldots, V_{n}^{*}\right)\right)$ is associated with a $\varphi$ (resp. $\psi$ ) Archimedean copula. Moreover, from the Marshall-Olkin algorithm described in Proposition $3.4, V_{1}, \ldots, V_{n}, \ldots$ and $V_{1}^{*}, \ldots, V_{n}^{*}, \ldots$ can be built as infinite sequences of exchangeable random variables. Let $D_{i}(\bar{p})=1_{\left\{V_{i} \leq \bar{p}\right\}}\left(\right.$ resp. $\left.D_{i}^{*}(\bar{p})=1_{\left\{V_{i}^{*} \leq \bar{p}\right\}}\right) i=1, \ldots, n, \ldots$ be the corresponding default indicator sequence. Since the supermodular order is closed under monotone transformations,

$$
\left(D_{1}(\bar{p}), \ldots, D_{n}(\bar{p})\right) \leq_{s m}\left(D_{1}^{*}(\bar{p}), \ldots, D_{n}^{*}(\bar{p})\right)
$$

holds for all $n$ and all $\bar{p}$. Thanks to Theorem 2.12, the associated mixture probabilities $\tilde{p}$ and $\tilde{p}^{*}$ are thus ordered in the convex order: $\tilde{p} \leq_{c x} \tilde{p}^{*}$ holds for all $\bar{p}$ in $[0,1]$. 
The previous framework includes several well-known parametric families of Archimedean copulas:

\begin{tabular}{|c|c|c|c|}
\hline Copula name & Generator $\varphi$ & Parameter & $V$-distribution \\
\hline Clayton & $t^{-\theta}-1$ & $\theta \geq 0$ & Gamma $(1 / \theta)$ \\
Gumbel & $(-\ln (t))^{\theta}$ & $\theta \geq 1$ & $\alpha$-Stable, $\alpha=1 / \theta$ \\
Franck & $-\ln \left[\left(1-e^{-\theta t}\right) /\left(1-e^{-\theta}\right)\right]$ & $\mathbb{R}^{*}$ & Logarithmic series \\
\hline
\end{tabular}

For each of these families, consider two parameters $\theta$ and $\theta^{*}$ associated with (resp.) generators $\varphi$ and $\phi$. It is easy to show that, if $\theta \leq \theta^{*}$, then $\varphi \circ \psi^{-1} \in \mathscr{L}_{\infty}^{*}$ (see Joe (1997) for more details). Therefore, when $\theta \leq \theta^{*}$ the corresponding mixture probabilities are ordered with respect to the convex order, $\tilde{p} \leq_{c x} \tilde{p}^{*}$, and $\left(D_{1}, \ldots, D_{n}\right) \leq_{s m}\left(D_{1}^{*}, \ldots, D_{n}^{*}\right)$.

We compute below (see Fig. 3) the mixture distribution functions associated with the Clayton copula $\left(F_{\tilde{p}}(p)=P(\tilde{p} \leq p)\right)$ for different levels of the dependence parameter $\theta$. In fact, when $\theta$ increases, the mixture distribution functions are ordered with respect to the less dangerous order (which is stronger than the convex order).

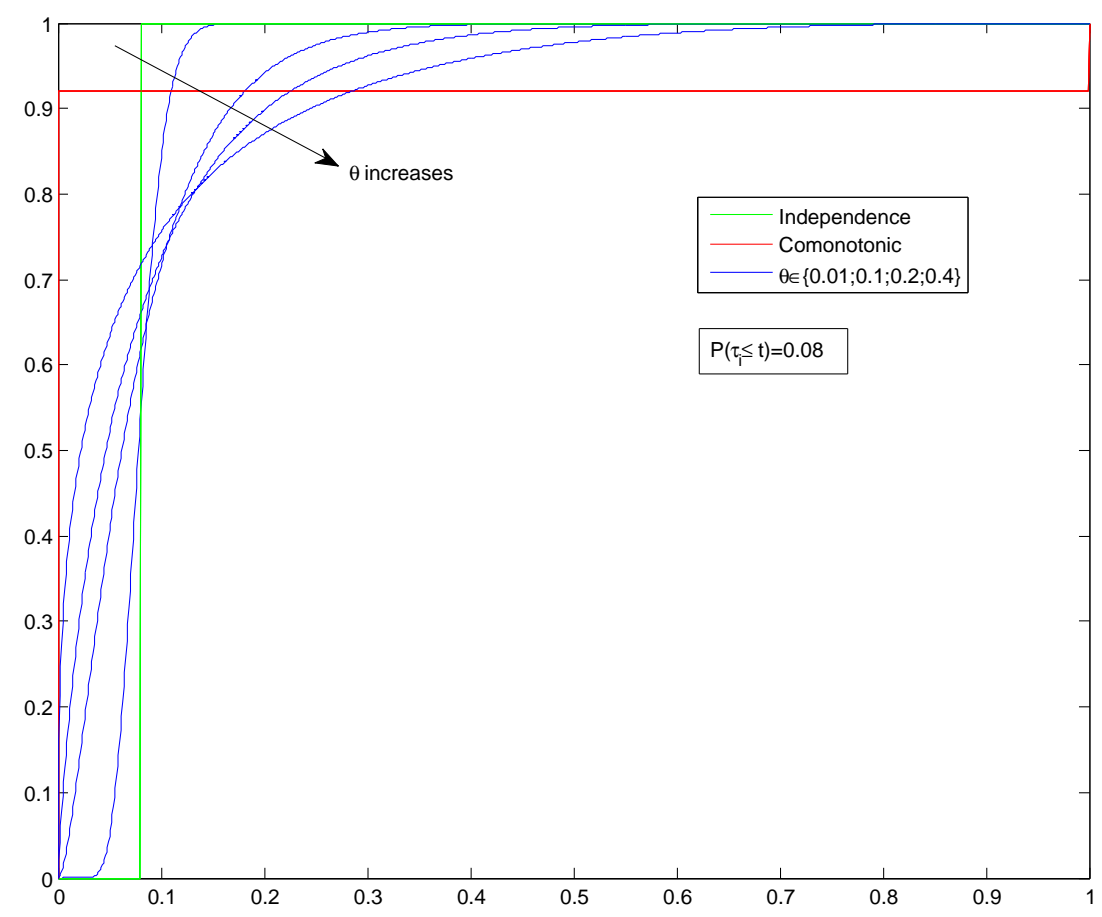

Fig. 3. Mixture distribution functions of the Clayton copula 


\subsection{Multivariate Poisson models}

These models originate from the theory of reliability where they are also called shock models. In multivariate Poisson models, default times correspond to the first jump instants of a multivariate Poisson process $\left(N_{t}^{1}, \ldots, N_{t}^{n}\right)$. We thereafter restrict the common Poisson shock model studied by Lindskog and McNeil (2003) and Elouerkhaoui (2006) to the case of two independent shocks. Each default can be triggered either by an idiosyncratic fatal shock or by a systemic non necessarily fatal shock. The Poisson process which drives a default event for name $i$ is expressed as:

$$
N_{t}^{i}=\bar{N}_{t}^{i}+\sum_{j=1}^{N_{t}} B_{j}^{i},
$$

where $\bar{N}_{t}^{i}$ and $N_{t}$ follow independent Poisson processes with (resp.) parameters $\bar{\lambda}$ and $\lambda$. We assume further that $\left(B_{j}^{i}\right)_{i, j}$ are iid Bernoulli random variables with mean $p$, independent of $N_{t}$ and $\bar{N}_{t}^{i}$. We can see that the background event (new jump of $N_{t}$ ) affects each name with a probability $p$. Default times are then described by:

$$
\tau^{i}=\inf \left\{t>0 \mid N_{t}^{i}>0\right\}, \quad i=1, \ldots, n .
$$

As $N_{t}^{i}$ is a Poisson process with parameter $\bar{\lambda}+p \lambda$, default dates $\tau^{i}, i=1, \ldots, n$ are then exponentially distributed with the same parameter. The dependence structure of the vector $\left(\tau_{1}, \ldots, \tau_{n}\right)$ is the Marshall-Olkin copula (See Elouerkhaoui (2006) for details about the copula function).

If $D_{i}=1_{\left\{\tau_{i} \leq t\right\}}, i=1 \ldots n$ denote the default indicators at date $t$, then $\left(D_{1}, \ldots, D_{n}\right)$ is an exchangeable Bernoulli random vector. Furthermore, $D_{1}, \ldots, D_{n}$ are independent given the common factor $N_{t}$. We aim at analyzing at a fixed horizon $t$, the influence of the risk parameters $(\bar{\lambda}, p, \lambda)$ on comparisons of $\left(D_{1}, \ldots, D_{n}\right)$ with respect to the supermodular order.

Thanks to remark (2.3), the mixture probability $\tilde{p}$ associated with $\left(D_{1}, \ldots, D_{n}\right)$ is equal to the conditional default probability $P\left(\tau_{i} \leq t \mid N_{t}\right)$. It is straightforward to derive the expression of $\tilde{p}$ and its distribution function:

$$
\begin{gathered}
\tilde{p}=P\left(\tau_{i} \leq t \mid N_{t}\right)=1-(1-p)^{N_{t}} \exp (-\bar{\lambda} t), \\
F(q)=P(\tilde{p} \leq q)=\sum_{n=0}^{n_{0}} \frac{(\lambda t)^{n}}{n !} \exp (-\lambda t)
\end{gathered}
$$

with

$$
n_{0}=\left[\frac{\bar{\lambda} t+\ln (1-q)}{\ln (1-p)}\right]
$$

Supermodular comparisons can only be performed between two random vectors with the same marginal distributions. Hence, if we denote by $(\bar{\lambda}, p, \lambda)$ and $\left(\bar{\lambda}^{*}, p^{*}, \lambda^{*}\right)$ the parameter sets corresponding to $\left(D_{1}, \ldots, D_{n}\right)$ and $\left(D_{1}^{*}, \ldots, D_{n}^{*}\right)$, the following equality must be satisfied:

$$
\bar{\lambda}+p \lambda=\bar{\lambda}^{*}+p^{*} \lambda^{*}
$$

Therefore, three directions can be explored depending on which parameter remains constant. 
(1) $\bar{\lambda}^{*}=\bar{\lambda}, p^{*} \geq p$ and $\lambda^{*} \leq \lambda$ : relative impact on the dependence structure of the two systemic parameters.

(2) $p^{*}=p, \lambda^{*} \geq \lambda$ and $\bar{\lambda}^{*} \leq \bar{\lambda}$ : comparison of systemic and idiosyncratic intensities.

(3) $\lambda^{*}=\lambda, p^{*} \geq p$ and $\bar{\lambda}^{*} \leq \bar{\lambda}$ : comparison of the probability of surviving a systemic shock and the idiosyncratic intensity.

For all these comparisons, we will see that $\tilde{p} \leq_{c x} \tilde{p}^{*}$ and $\left(D_{1}, \ldots, D_{n}\right) \leq_{s m}\left(D_{1}^{*}, \ldots, D_{n}^{*}\right)$. The supermodular order results for framework (2) and (3) are quite expectable because comparisons are performed between the idiosyncratic parameter and one of the common shock parameters which, in fact govern the dependence within the default indicator vector. They just reflect the fact that an increase of the common shock parameter $\lambda$ (resp. $p$ ) yields an increase of the dependence in the sense of the supermodular order. The result for framework (1) is much less intuitive than the two others since the comparison is performed between the two parameters $(p$ and $\lambda$ ) governing the dependence. The probability $p$ that a systemic shock turns out to be fatal actually has a larger impact on the dependence structure than the intensity $\lambda$ of this systemic shock.

\section{Proposition 3.6 Comparison 1}

Let parameter sets $(\bar{\lambda}, \lambda, p)$ and $\left(\bar{\lambda}^{*}, \lambda^{*}, p^{*}\right)$ be such that $\bar{\lambda}+p \lambda=\bar{\lambda}^{*}+p^{*} \lambda^{*}$ and assume that $\bar{\lambda}^{*}=\bar{\lambda}, p^{*} \geq p$ and $\lambda^{*} \leq \lambda$. Let us consider $\tilde{p}=1-(1-p)^{N_{t}} \exp (-\bar{\lambda} t)$ and $\tilde{p}^{*}=1-(1-$ $\left.p^{*}\right)^{N_{t}^{*}} \exp (-\bar{\lambda} t)$, the corresponding mixture probabilities. Then:

$$
\tilde{p} \leq_{c x} \tilde{p}^{*}
$$

Proof: There is a real number $1 \leq \alpha \leq 1 / p$ such that $p^{*}=\alpha p$ and $\lambda^{*}=\lambda / \alpha$. As the convex order is stable by linear transformations, we need to prove that $(1-p)^{N_{t}} \leq_{c x}\left(1-p^{*}\right)^{N_{t}^{*}}$. As $N_{t}^{*}$ is a Poisson process with parameter $\lambda / \alpha$, we have:

$$
N_{t}^{*} \stackrel{d}{=} \sum_{i=1}^{N_{t}} B_{i}
$$

where $\left(B_{i}\right)_{i \geq 1}$ forms a random sequence of independent Bernoulli random variables with parameter $1 / \alpha$, independent of $N_{t}$. Let us remark that:

$$
E\left[\left(1-p^{*}\right)^{\sum_{i=1}^{N_{t}} B_{i}} \mid N_{t}\right]=\prod_{i=1}^{N_{t}} E\left[(1-\alpha p)^{B_{i}}\right]=(1-p)^{N_{t}},
$$

which ends the proof, thanks to Strassen's theorem.

\section{Proposition 3.7 Comparison 2}

Let parameter sets $(\bar{\lambda}, \lambda, p)$ and $\left(\bar{\lambda}^{*}, \lambda^{*}, p^{*}\right)$ be such that $\bar{\lambda}+p \lambda=\bar{\lambda}^{*}+p^{*} \lambda^{*}$ and assume that $p^{*}=$ $p, \lambda^{*} \geq \lambda$ and $\bar{\lambda}^{*} \leq \bar{\lambda}$. Let us consider $\tilde{p}=1-(1-p)^{N_{t}} \exp (-\bar{\lambda} t)$ and $\tilde{p}^{*}=1-(1-p)^{N_{t}^{*}} \exp \left(-\bar{\lambda}^{*} t\right)$, the corresponding mixture probabilities. Then:

$$
\tilde{p} \leq_{c x} \tilde{p}^{*}
$$


Proof: There is a real number $\alpha \geq 0$ such that $\lambda^{*}=\lambda+\alpha$ and $\bar{\lambda}^{*}=\bar{\lambda}-\alpha p$. As the convex order is stable by linear transformations, we need to prove that $(1-p)^{N_{t}} \exp (-\bar{\lambda} t) \leq_{c x}(1-$ $p)^{N_{t}^{*}} \exp \left(-\bar{\lambda}^{*} t\right)$. As $N_{t}^{*}$ is a Poisson process with parameter $\lambda+\alpha$, there exists a Poisson process $\tilde{N}_{t}$ with parameter $\alpha$, independent of $N_{t}$ and such that $N_{t}^{*} \stackrel{d}{=} N_{t}+\tilde{N}_{t}$. But, as $E\left[(1-p)^{\tilde{N}_{t}}\right]=$ $\exp (-\alpha p t)$, we obtain:

$$
E\left[(1-p)^{N_{t}+\tilde{N}_{t}} \exp (-(\bar{\lambda}-\alpha p) t) \mid N_{t}\right]=(1-p)^{N_{t}} \exp (-\bar{\lambda} t)
$$

which concludes the proof, thanks to Strassen's theorem.

\section{Proposition 3.8 Comparison 3}

Let parameter sets $(\bar{\lambda}, \lambda, p)$ and $\left(\bar{\lambda}^{*}, \lambda^{*}, p^{*}\right)$ be such that $\bar{\lambda}+p \lambda=\bar{\lambda}^{*}+p^{*} \lambda^{*}$ and assume that $\lambda^{*}=$ $\lambda, p^{*} \geq p$ and $\bar{\lambda}^{*} \leq \bar{\lambda}$. Let us consider $\tilde{p}=1-(1-p)^{N_{t}} \exp (-\bar{\lambda} t)$ and $\tilde{p}^{*}=1-\left(1-p^{*}\right)^{N_{t}} \exp \left(-\bar{\lambda}^{*} t\right)$, the corresponding mixture probabilities. Then:

$$
\tilde{p} \leq_{D} \tilde{p}^{*}
$$

Proof: Let us define $\alpha$ by $\alpha=p^{*}-p$; then $\bar{\lambda}^{*}=\bar{\lambda}-\alpha \lambda$. As for the additive copula framework, let us show that the mixture distributions are ordered with respect to the less dangerous order. In view of the expression of $F$ (equation 3.13), it is straightforward to study the sign of $F-F^{*}$ which only depends on the behaviour of $n_{0}-n_{0}^{*}$ with respect to $q$. Therefore, it remains to find the values of $q$ for which the following inequality holds:

$$
\frac{\bar{\lambda} t+\ln (1-q)}{\ln (1-p)} \leq \frac{(\bar{\lambda}-\alpha \lambda) t+\ln (1-q)}{\ln (1-(p+\alpha))}
$$

It follows that:

$$
q \leq q_{0}=1-\exp \left(-\frac{a}{b} t\right) \text { with: }
$$

$a=[\ln (1-p)-\ln (1-(p+\alpha))] \bar{\lambda}-\alpha \lambda \ln (1-p) \geq 0$ and $b=\ln \left(\frac{1-p}{1-(p+\alpha)}\right) \geq 0$. We have thus shown that it exists $q_{0} \in[0,1]$ such that if $q<q_{0}$ then $F(q)-F^{*}(q) \leq 0$ and $F(q)-F^{*}(q) \geq 0$ otherwise. Besides, $E[\tilde{p}]=E\left[\tilde{p}^{*}\right]=1-\exp (-(\bar{\lambda}+p \lambda) t)$, eventually shows that $\tilde{p} \leq_{c x} \tilde{p}^{*}$.

As an example (see Fig. 4), let us consider two Poisson models with parameter set $(\bar{\lambda}, \lambda, p)$ and $\left(\bar{\lambda}^{*}, \lambda^{*}, p^{*}\right)$ as in Proposition 3.8 .

As expected, the distribution function of $\tilde{p}(p=0.1)$ is less dangerous than the one of $\tilde{p}^{*}(p=0.8)$.

\subsection{Supermodular order and structural models}

In this section, we show that default indicators in a simple structural framework are ordered with respect to the supermodular order when the dependence between the assets of the corresponding 


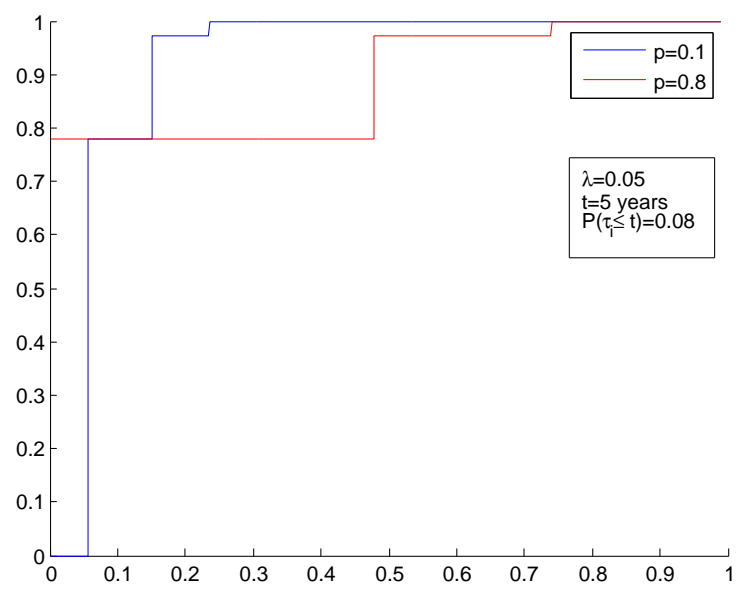

Fig. 4. Mixture distribution functions of the Multivariate Poisson model

firms increases. We are concerned with $n$ firms which may default in a period $[0, T]$. Their asset dynamics $X^{1}, \ldots, X^{n}$ are simply expressed as $n$ correlated Brownian motions:

$$
X_{t}^{i}=\rho W_{t}+\sqrt{1-\rho^{2}} W_{t}^{i}, \quad i=1 \ldots n,
$$

where $W, W^{i}, i=1, \ldots n$ are independent standard Wiener processes.

Default of firm $i$ is triggered when the process $X^{i}$ falls below a constant barrier $a_{i}$ for the first time. The corresponding default date $\tau_{i}$ is expressed as:

$$
\tau_{i}=\inf \left\{t \in \mathbb{R}^{+} \mid X_{t}^{i} \leq a_{i}\right\}, \quad i=1 \ldots n
$$

This framework has been investigated by Hull et al. (2005) with some emphasis on the pricing of CDOs. We want to show that an increase of the dependence parameter $\rho$ yields an increase of the default indicators dependence with respect to the supermodular order.

Proposition 3.9 Let $\tau_{i}$ (resp. $\left.\tau_{i}^{*}\right), i=1, \ldots n$, be the default dates of $n$ firms with asset dependence parameter $\rho$ (resp. $\left.\rho^{*}\right)$ in the structural framework described above. For any fixed time horizon $T$, let us define by $D_{i}=1_{\left\{\tau_{i} \leq T\right\}}$ (resp. $D_{i}^{*}=1_{\left\{\tau_{i}^{*} \leq T\right\}}$ ), $i=1, \ldots n$, the corresponding default indicators. If $\rho \leq \rho^{*}$, then:

$$
\left(D_{1}, \ldots, D_{n}\right) \leq_{s m}\left(D_{1}^{*}, \ldots, D_{n}^{*}\right)
$$

Proof: Let $\left(X_{t}^{1}, \ldots, X_{t}^{d}\right)$ (resp. $\left.\left(Y_{t}^{1}, \ldots, Y_{t}^{d}\right)\right)$ be the vector of asset values at time $t$ associated with the dependence parameter $\rho$ (resp. $\rho^{*}$ ). As the event $\left\{\tau_{i} \leq T\right\}$ (resp. $\left\{\tau_{i}^{*} \leq T\right\}$ ) is equal to $\left\{\min \left\{X_{t}^{i} \mid t \in[0, T]\right\} \leq a_{i}\right\}$ (resp. $\left\{\min \left\{Y_{t}^{i} \mid t \in[0, T]\right\} \leq a_{i}\right\}$ ), the default indicators $D_{i}$ (resp. $\left.D_{i}^{*}\right) i=1, \ldots n$, are non increasing functions of their corresponding asset value minimums on $[0, T]$. In view of the stability property of the supermodular order with respect to monotone transformations, we would like to show that when $\rho \leq \rho^{*}$, then:

$$
\left(\min _{t \in[0, T]} X_{t}^{1}, \ldots, \min _{t \in[0, T]} X_{t}^{d}\right) \leq_{s m}\left(\min _{t \in[0, T]} Y_{t}^{1}, \ldots, \min _{t \in[0, T]} Y_{t}^{d}\right)
$$


It is well known that two Gaussian vectors with the same marginals can be compared with respect to the supermodular order if their correlation matrices are componentwise ordered (see for example Theorem 3.13.5 in Müller and Stoyan (2002)). This is the case in our simple framework. When $\rho \leq \rho^{*}$, the asset value vectors at time $t$ are comparable for all $t$ :

$$
\left(X_{t}^{1}, \ldots, X_{t}^{d}\right) \leq_{s m}\left(Y_{t}^{1}, \ldots, Y_{t}^{d}\right)
$$

We can now complete the proof of Proposition 3.9 using the following lemmas.

Lemma 3.10 Let us consider four independent $\mathbb{R}^{d}$ valued random vectors $\mathbf{X}_{1}, \mathbf{X}_{2}, \mathbf{Y}_{1}, \mathbf{Y}_{2}$ and define the function $\varphi: \mathbb{R}^{d} \times \mathbb{R}^{d} \rightarrow \mathbb{R}^{d}$ as:

$$
\varphi(\mathbf{X}, \mathbf{Y})=\left(X^{1}+\min \left(Y^{1}, 0\right), \ldots, X^{d}+\min \left(Y^{d}, 0\right)\right)
$$

Assume that $\mathbf{X}_{1} \leq_{s m} \mathbf{Y}_{1}$ and $\mathbf{X}_{2} \leq_{s m} \mathbf{Y}_{2}$, then $\varphi\left(\mathbf{X}_{1}, \mathbf{X}_{2}\right) \leq_{s m} \varphi\left(\mathbf{Y}_{1}, \mathbf{Y}_{2}\right)$.

Proof: By invariance of the supermodular order with respect to monotone transformations,

$$
\left(X_{1}^{1}+\alpha_{1}, \ldots, X_{1}^{d}+\alpha_{n}\right) \leq_{s m}\left(Y_{1}^{1}+\alpha_{1}, \ldots, Y_{1}^{d}+\alpha_{n}\right)
$$

and

$$
\left(\beta_{1}+\min \left(X_{2}^{1}, 0\right), \ldots, \beta_{n}+\min \left(X_{2}^{d}, 0\right)\right) \leq_{s m}\left(\beta_{1}+\min \left(Y_{2}^{1}, 0\right), \ldots, \beta_{n}+\min \left(Y_{2}^{d}, 0\right)\right)
$$

hold for all real vectors $\left(\alpha_{1}, \ldots, \alpha_{n}\right)$ and $\left(\beta_{1}, \ldots, \beta_{n}\right)$. By closure of the supermodular order under mixture and by independence of $\mathbf{X}_{1}, \mathbf{Y}_{1}, \min \left(\mathbf{X}_{2}, 0\right)$ and $\min \left(\mathbf{Y}_{2}, 0\right)$, we obtain:

$$
\varphi\left(\mathbf{X}_{1}, \mathbf{X}_{2}\right) \leq_{s m}\left(Y_{1}^{1}+\min \left(X_{2}^{1}, 0\right), \ldots, Y_{1}^{d}+\min \left(X_{2}^{d}, 0\right)\right)
$$

and

$$
\left(Y_{1}^{1}+\min \left(X_{2}^{1}, 0\right), \ldots, Y_{1}^{d}+\min \left(X_{2}^{d}, 0\right)\right) \leq_{s m} \varphi\left(\mathbf{Y}_{1}, \mathbf{Y}_{2}\right)
$$

Thanks to the transitivity of the supermodular order, (3.25) and (3.26) yields the desired result.

Lemma 3.11 Let $T$ be a fixed horizon date. Let $\mathbf{X}$ and $\mathbf{Y}$ be two independent continuous $\mathbb{R}^{d}$ valued processes with stationary independent increments and such that for all $t$ in $[0, T]$ :

$$
\mathbf{X}_{t} \leq_{s m} \mathbf{Y}_{t}
$$

Denote by:

$$
M^{X}=\left(\min _{t \in[0, T]} X_{t}^{1}, \ldots, \min _{t \in[0, T]} X_{t}^{d}\right)
$$

and:

$$
M^{Y}=\left(\min _{t \in[0, T]} Y_{t}^{1}, \ldots, \min _{t \in[0, T]} Y_{t}^{d}\right)
$$

Then,

$$
M^{X} \leq_{s m} M^{Y}
$$


Proof: First, let us restrict the interval $[0, T]$ to a finite subdivision $S_{n}=\left(t_{0}=0, t_{1}, \ldots, t_{n}=T\right)$. Let us define:

$$
M_{n}^{X}=\left(\min _{t_{i} \in S_{n}} X_{t_{i}}^{1}, \ldots, \min _{t_{i} \in S_{n}} X_{t_{i}}^{d}\right),
$$

and:

$$
M_{n}^{Y}=\left(\min _{t_{i} \in S_{n}} Y_{t_{i}}^{1}, \ldots, \min _{t_{i} \in S_{n}} Y_{t_{i}}^{d}\right)
$$

We will first show that for all $n \geq 2, M_{n}^{X} \leq_{s m} M_{n}^{Y}$. Denote by $\Delta \mathbf{X}_{t_{i}}=\mathbf{X}_{t_{i}}-\mathbf{X}_{t_{i-1}}$, (resp. $\Delta \mathbf{Y}_{t_{i}}=\mathbf{Y}_{t_{i}}-\mathbf{Y}_{t_{i-1}}$ ), $i=1, \ldots, n$ the increments of $\mathbf{X}$, (resp. $\mathbf{Y}$ ) on $S_{n}$. Let us assume that $\mathbf{X}$ and $\mathbf{Y}$ start from 0. It is possible to write $M_{n}^{X}$ (resp. $M_{n}^{Y}$ ) as a function of the $\Delta \mathbf{X}_{t_{i}}$ 's (resp. $\left.\Delta \mathbf{Y}_{t_{i}}{ }^{\prime} \mathrm{s}\right), i=1, \ldots, n$ :

$$
\begin{aligned}
M_{n}^{X} & =\Delta \mathbf{X}_{t_{1}}+\min \left(\Delta \mathbf{X}_{t_{2}}+\min \left(\Delta \mathbf{X}_{t_{3}}+\ldots+\min \left(\Delta \mathbf{X}_{t_{n}}, 0\right), \ldots, 0\right), 0\right) \\
& =\varphi\left(\Delta \mathbf{X}_{t_{1}}, \varphi\left(\ldots, \varphi\left(\Delta \mathbf{X}_{t_{n-1}}, \Delta \mathbf{X}_{t_{n}}\right)\right)\right)
\end{aligned}
$$

and:

$$
\begin{aligned}
M_{n}^{Y} & =\Delta \mathbf{Y}_{t_{1}}+\min \left(\Delta \mathbf{Y}_{t_{2}}+\min \left(\Delta \mathbf{Y}_{t_{3}}+\ldots+\min \left(\Delta \mathbf{Y}_{t_{n}}, 0\right), \ldots, 0\right), 0\right) \\
& =\varphi\left(\Delta \mathbf{Y}_{t_{1}}, \varphi\left(\ldots, \varphi\left(\Delta \mathbf{Y}_{t_{n-1}}, \Delta \mathbf{Y}_{t_{n}}\right)\right)\right)
\end{aligned}
$$

where $\varphi$ is defined as (3.24).

Since $\mathbf{X}$ and $\mathbf{Y}$ are independent $\mathbb{R}^{d}$ processes with stationary independent increments, for all $i=1, \ldots, n, \Delta \mathbf{X}_{t_{i}} \stackrel{d}{=} \mathbf{X}_{t_{i}-t_{i-1}} \leq_{s m} \mathbf{Y}_{t_{i}-t_{i-1}} \stackrel{d}{=} \Delta \mathbf{Y}_{t_{i}}$ and for all $i \neq j, \Delta \mathbf{X}_{t_{i}}, \Delta \mathbf{X}_{t_{j}}$, $\Delta \mathbf{Y}_{t_{i}}$ and $\Delta \mathbf{Y}_{t_{j}}$ are independent random vectors. Hence, for all $k=2, \ldots, n-1, \Delta \mathbf{X}_{t_{k-1}}$, $\varphi\left(\Delta \mathbf{X}_{t_{k}}, \varphi\left(\ldots, \varphi\left(\Delta \mathbf{X}_{t_{n-1}}, \Delta \mathbf{X}_{t_{n}}\right)\right)\right), \Delta \mathbf{Y}_{t_{k-1}}$ and $\varphi\left(\Delta \mathbf{Y}_{t_{k}}, \varphi\left(\ldots, \varphi\left(\Delta \mathbf{Y}_{t_{n-1}}, \Delta \mathbf{Y}_{t_{n}}\right)\right)\right)$ are independent. Thus, by successive uses of Lemma 3.10, we obtain for all $n$ :

$$
M_{n}^{X} \leq_{s m} M_{n}^{Y}
$$

Let us now consider $S_{n}=\left(t_{0}^{n}=0, \ldots, t_{n}^{n}, t_{n+1}^{n}=T\right)$ as a sequence of $[0, T]$-subdivisions whose mesh tends to zero. As $\mathbf{X}$ and $\mathbf{Y}$ are continuous processes, then for all $\omega \in \Omega$ :

$$
M_{n}^{X}(\omega) \underset{n \rightarrow \infty}{\longrightarrow} M^{X}(\omega),
$$

and:

$$
M_{n}^{Y}(\omega) \underset{n \rightarrow \infty}{\longrightarrow} M^{Y}(\omega)
$$

This obviously implies convergence in distribution and allows to conclude thanks to the stability property of the supermodular order with respect to weak convergence.

Eventually, Proposition 3.9 immediately derives from Lemma 3.11. Let us remark that this result is preserved when the threshold is a linear function of time, say $\phi(t)=a t+b$, since default dates can be expressed as:

$$
\tau_{X}=\inf \left\{t \in \mathbb{R}^{+} \mid X_{t}-(a t+b) \leq 0\right\},
$$


and this new specification neither affects the Gaussian dependence structure nor the stationary and independent increments property required here. Moreover, the stationarity assumption can also be relaxed easily.

In our example, supermodular order comparisons of default indicator vectors have not been derived from the convex ordering of associated mixture distributions since these cannot be expressed analytically in this context. But it is possible to estimate the mixture distribution function in the previous structural framework using Monte Carlo simulations. We have drawn in Fig. 5 the mixture distributions associated with two levels of the dependence parameter $\rho$.

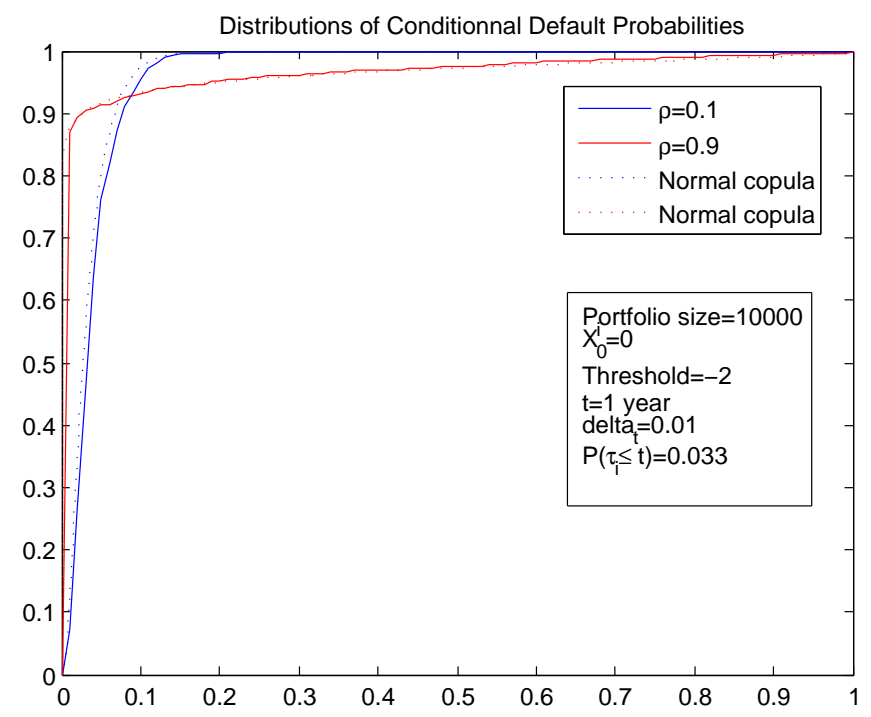

Fig. 5. Distributions of Conditional Default Probabilities

We can observe that an increase of $\rho$ yields an increase of the dangerousness of the corresponding mixture distributions in the sense of the less dangerous order. We additionally drew mixture distributions obtained from a Gaussian copula model for the same values of $\rho$. The default probability $\bar{p}=P\left(\tau_{i} \leq t\right)$ has been chosen so that that it corresponds to the one of the structural model. We can remark that computed distributions are very similar for a fixed $\rho$ which is coherent with the result of Hull et al. (2005) where CDO tranche premiums are found to be very close for the two frameworks.

Let us also remark that (3.22) also holds for any fixed time horizon $T$ which is sufficient when considering applications developed in the paper. For example, comparisons between CDO tranche premiums can be derived since they only involve the marginal distributions of the aggregate loss for several horizons. However, thanks to the closure property of the supermodular order under mixture, (3.22) still holds when $T$ is a stopping time with respect to a specific filtration independent from the Brownians filtrations (generated by $W$ and $W^{i}, i=1, \ldots, n$ ). This cannot be extended to any stopping times. Indeed, let us compare two bivariate vectors of default indicators $\left(D_{1}, D_{2}\right)$ and $\left(D_{1}^{*}, D_{2}^{*}\right)$ defined as in Proposition 3.9. We assume that $T=\tau_{1} . T$ is thus a stopping time with respect to the filtration generated by $W$ and $W^{1}$. But $\left(D_{1}, D_{2}\right)=$ 
$\left(1,1_{\left\{\tau_{2} \leq \tau_{1}\right\}}\right)$ and $\left(D_{1}^{*}, D_{2}^{*}\right)=\left(1_{\left\{\tau_{1}^{*} \leq \tau_{1}\right\}}, 1_{\left\{\tau_{2}^{*} \leq \tau_{1}\right\}}\right)$ cannot be compared anymore as supermodular order comparisons require marginals to be equal.

\section{Conclusion}

In our framework, default indicators form an exchangeable sequence of Bernoulli random variables. Capitalizing on de Finetti's theorem, we could relate the supermodular ordering of default indicator vectors to the convex ordering of the corresponding mixture probabilities. As a consequence, we performed a unified analysis of dependence for additive factor copula, Archimedean copula, multivariate Poisson and structural models.

\section{References}

Bäuerle, N., 1997. Inequalities for stochastic models via supermodular orderings. Communications in Statistics, Stochastic Models 13, 181-201.

Bäuerle, N., Müller, A., 1998. Modeling and comparing dependencies in multivariate risk portfolios. ASTIN Bulletin 28, 59-76.

Bäuerle, N., Müller, A., 2005. Stochastic orders and risk measures: consistency and bounds. Insurance: Mathematics and Economics 38, 132-148.

Burgert, C., Rüschendorf, L., 2006. Consistent risk measures for portfolio vectors. Insurance: Mathematics and Economics 38, 289-297.

Burtschell, X., Gregory, J., Laurent, J.-P., 2005. A comparative analysis of CDO pricing models, working Paper, ISFA Actuarial School, University of Lyon and BNP-Paribas.

Burtschell, X., Gregory, J., Laurent, J.-P., 2007. Beyond the Gaussian copula: stochastic and local correlation. Journal of Credit Risk 3 (1), 31-62.

Cossette, H., Gaillardetz, P., Marceau, E., Rioux, J., 2002. On two dependent individual risk models. Insurance: Mathematics and Economics 30 (2), 153-166.

Denuit, M., Dhaene, J., Goovaerts, M., Kaas, R., 2005. Actuarial Theory for Dependent Risks Measures, Orders and Models. Wiley.

Denuit, M., Frostig, E., June 2007. Comparison of dependence in factor models with application to credit risk portfolios, working paper.

Denuit, M., Müller, A., 2002. Smooth generators of integral stochastic orders. The Annals of Applied Probability 12 (4), 1174-1184.

Dhaene, J., Goovaerts, M., 1997. On the dependency of risks in the individual life model. Insurance: Mathematics and Economics 19, 243-253.

Elouerkhaoui, Y., 2006. Etude des Problèmes de Corrélation et d'Incomplétude dans les Marchés de Crédit. Phd thesis, University of Paris Dauphine.

Guegan, D., Houdain, J., 2005. Collateralized debt obligations pricing and factor models: a new methodology using Normal Inverse Gaussian distributions.

Hull, J., Predescu, M., White, A., 2005. The valuation of correlation-dependent credit derivatives using a structural model, working paper, University of Toronto.

Hull, J., White, A., Winter 2004. Valuation of a CDO and $n^{\text {th }}$ to default CDS without Monte Carlo simulation. The Journal of Derivatives 12 (2), 8-23. 
Jaynes, E., 1986. Some applications and extensions of the de Finetti representation theorem. Bayesian Inference and Decision Techniques, 31-42.

Joe, H., 1997. Multivariate Models and Dependence Concepts. Chapman and Hall, Monographs on Statistics and Applied Probability, London.

Kalemanova, A., Schmid, B., Werner, R., Spring 2007. The Normal Inverse Gaussian distribution for synthetic CDO pricing. The Journal of Derivatives.

Kendall, D., 1967. On finite and infinite sequences of exchangeable events. Studia Sci. Math. Hung. 2, 319-327.

Laurent, J.-P., Gregory, J., 2005. Basket default swaps, CDOs and factor copulas. Journal of Risk 7 (4), 103-122.

Lefèvre, C., Utev, S., 1996. Comparing sums of exchangeable Bernoulli random variables. Journal of Applied Probability 33, 285-310.

Lindskog, F., McNeil, A., 2003. Common Poisson shock models: applications to insurance and credit risk modelling. ASTIN Bulletin 33 (2), 209-238.

Marshall, A. W., Olkin, I., 1988. Families of multivariate distributions. Journal of the American Statistical Association 83, 834-841.

Moosbrucker, T., 2006. Pricing CDOs with correlated Variance Gamma distributions, working paper, Centre for Financial Research, University of Cologne.

Müller, A., 1997. Stop-loss order for portfolios of dependent risks. Insurance: Mathematics and Economics 21, 219-223.

Müller, A., Scarsini, M., 2000. Some remarks on the supermodular order. Journal of Multivariate Analysis 73, 107-119.

Müller, A., Stoyan, D., 2002. Comparison Methods for Stochastic Models and Risks. John Wiley \& Sons Ltd., Chichester Wiley Series in Probability and Statistics.

Nelsen, R., 1999. An Introduction to Copulas. Springer, New York.

Shaked, M., Shanthikumar, J., 1994. Stochastic Orders and Their Applications. Academic Press, London.

Wei, G., Hu, T., 2002. Supermodular dependence ordering on a class of multivariate copulas. Statistics and Probability Letters 57, 357-385. 